\title{
Iron Hematoxylin Staining Method
}

National Cancer Institute

\section{Source}

National Cancer Institute. Iron Hematoxylin Staining Method. NCI Thesaurus. Code C85650.

A microscopy staining method that utilizes a mixture of iron and hematoxylin to visualize intracellular structures in a microscopic sample. In this dye system, iron acts as a mordant to hematoxylin rendering it insoluble, thereby allowing irreversible blue-black colored dyeing to the cell nucleus. 